including tularemia, botulism, and brucellosis.

Antibiotics can be used both before exposure and immediately after exposure to prevent disease caused by sensitive bacteria, but are ineffective against organisms that are naturally or artificially resistant to them. Although using antibiotics for long-term prophylaxis is unrealistic because of the cost, logistic burden, and side effects, good results can be used if they are given shortly after exposure if an attack has been identified. Currently, quinolones such as ciprofloxacin are recommended widely for prophylaxis and therapy of plague and anthrax. There are very few useful antiviral agents, but cidofovir may be useful against smallpox. Generic immunomodulators can have a wide spectrum of activity, but are likely to be of limited utility because of side effects.

Keywords: anthrax; antibiotics; antiviral agents; attack; bacteria; bruccelosis; botulism; cost; immunomodulators; plaque; smallpox; terrorism; tularemia; vaccination; vaccines; viral; warfare, biological

Prehosp Disast Med 2003:18:s(1)s11.

\section{Free Topics}

\section{The International Health Specialist Program}

\section{Lieut. Col. Eugene Bonventre}

United States Air Force

International security challenges have changed significantly in the past decade. The bipolar world no longer exists; threats present themselves from many different sources, often as a result of a clash between different cultures. According to the Chief of Staff of the United States (U.S.) Air Force, "our expeditionary force requires airmen with international insight, foreign language proficiency, and cultural understanding."

The International Health Specialist (IHS) Program was created in 2001 as a cadre of medical professionals who possess language and cultural skills matched to military needs, to facilitate relationships with international civilian and military partners. The IHS personnel establish contacts with the Red Cross Movement and key United Nations agencies. They coordinate their efforts with nongovernmental organizations (NGOs), being careful not to violate the NGO's neutrality. They coordinate U.S. participation in exercises, such as MEDCEUR in the Baltics and Central and Eastern Europe, and MEDFLAG in Africa. The IHS program accepts all medical specialties, but members must maintain currency and competency in their primary medical specialty.

The European IHS team is based at the Ramstein Air Base in Germany. Two recent missions will be discussed. In Uganda, IHS interfaced with UNAIDS and the World Health Organization (WHO) to provide medical care in remote villages, and organized outcome studies to demonstrate their efficacy. In Sierra Leone, the IHS helped to rebuild a medical system ravaged by civil war. Both missions required situation-specific cultural knowledge, training, and experience.

Cultural skills are critical in shaping world events. It is hoped that such "medical diplomats" will facilitate regional cooperation in potential conflict zones.

Keywords: culture; efficacy; exercises; International Health

Specialists; language; non-governmental organizations; Red

Cross; security; U.S. Air Force; war, civil; World Health

Organization

Prebosp Disast Med 2003:18:s(1)s11.

E-mail: eugene.bonventre@ramstein.af.mil

Organization-Methodic Problems of Established

Medical-Diagnostic Standards in Trans-Border

Situations

Prof. Victor N. Preobrazbensky

All-Russian Centre for Disaster Medicine ("Zaschita"), Moscow,

Russia

Russia's experience in emergency health relief, including at the international level (Turkey, Colombia, Afghanistan) shows the necessity of developing international standards to increase efficiency in rendering medical assistance. At the same time, analysis of the existing standards of medical provision at the prehospital stage in some European countries in chemical accidents relief shows the need for detailed consideration and comparison of main indices for the provision of medical evacuation. Thus, it is necessary to analyze the existing approaches to the provision of medical evacuation in trans-border emergencies using the following main trends: (1) principles of medical triage of the injured; (2) amount of medical assistance provided at the prehospital stage; and (3) principles of evacuation of the injured.

The international experience of ARCDM "Zaschita" in health relief following earthquakes shows that it is necessary to unify medical diagnostic tactics in field medical institutions on the diagnosis and treatment of post-traumatic shock, crush syndrome, etc., to form a complex of medical-preventive measures during evacuation of the injured with the given pathology, etc. Thus, for the provision of medical evacuation in emergency trans-border relief, it is necessary to form a working group with representatives from different countries to analyze existing experience and establish unified standards.

Keywords: aid; ARCDM; assistance; chemical events; diagnosis; earthquakes; evacuation; experience; organization; prevention; relief; standards; trans-border; triage; "Zaschita"

Prehosp Disast Med 2003:18:s(1)s11.

E-mail: rcdm.org@ru.net

\section{Emergency Medical Care in Mass Trauma and Methodology for Its Realization \\ Dr. George OrakhelashviIi}

Tbilisi State Medical University, Tbilisi, Georgia

Forecasting and prevention of catastrophic events and elimination of their consequences have become one of the major problems in the world. One of the most devastating events, the place and time of which is practically impossible to forecast, is an earthquake. However, the practice of organizing emergency medical care for the victims of an earthquake indicates that such care, as a rule, is improvised, delayed, and inefficient. Thus, the development of new complex strategies for organizing and improving intra- and inter-state systems for emergency medical care during disasters is essential.

Therefore, in the Department of Disaster Medicine, a 
new concept of regional emergency medical care in case of mass trauma and a methodology for its realization have been developed. They are based on the most rational and economically acceptable organizational, medical, and diagnostic principles and methods. Simple and safe methods for the provision of total intravenous anesthesia have been developed. These methods do not require the use of complex narcosis equipment and compressed gases. The anesthesia methods were applied to more than 1,200 victims with multiple trauma-induced injuries and/or crush-syndrome. Based on the author's experience and the models for rendering the emergency surgical care to the victims, it has been established that these general anesthesia methods increase the capacity of surgical brigades by a minimum of 30 victims per day. A transfusion therapy complex for treating victims with severe crush-syndrome also has been developed and tested experimentally. As the results of application of the experimental use of the new complexes, all of the animals survived as did the injured extremities following functional rehabilitation. If realized in the treatment of victims in mass casualty situations, use of the proposed concept for the functioning of a field hospital with 80 beds to treat victims with traumatic injuries based on the principle of availability of reasonably sufficient amounts of material and technical supplies and of medicines and personnel, would allow provision of highly efficient emergency medical care to 400 such victims per day. At the same time, temporary hospitalization of the victims would last 4-6 hours; and the safety of their transportation by road and by air would improve significantly. Regional centers that actually would be engaged in establishing and coordinating the services of emergency medical care in the countries members; in improving the readiness of these services for massive numbers of casualties; and in working out the planes of joint activities. This plan stipulates the timelimits and a list and succession of essential measures and actions that will be performed by the country suffering from the disaster and the countries providing the humanitarian aid to it, including the provision of mobile military and civil fields hospitals.

Conclusion: Only the described strategy can lead to real success, i.e., to a significant decrease in the lethality and numbers of complications among the disaster victims without utilization of difficult and expensive methods of treatment.

Keywords: anesthesia, field; disasters; earthquake; efficiency; field hospitals; medical care; plan; surgery; trauma; victims

Prehosp Disast Med 2003;18(s1)s12.

\section{Presentation of Armed Forces Health Service / Veterinary Service of SAF}

Major Brane Dolenc, dr.vet.med.

Ministry of Defense, Armed Forces Health Service / Veterinary Service of SAF, Slovenia

The Veterinary Service of the SAF has operated autonomously within Armed Forces Health Service since October 2001. The beginnings of Veterinary Service go back to the time just after the war for Slovenian independence in 1991, when m.sc. Peter Levstek began working in a Canine unit. Four NCO-veterinary technicians and four
CO-Doctors of Veterinary Medicine work in the Veterinary Service of the SAF. They obtain ongoing continuing education in Slovenia and abroad, so they can continue to be highly qualified for completing all tasks for which they are responsible.

Beginning in 2000, the Veterinary Service of the SAF has combined the activities four units: (1) A military working dogs healthcare unit; (2) A unit for epizoology; (3) A unit for food hygiene; and (4) A unit for the breeding and training of military working dogs. The Veterinary Service of the SAF cooperates closely with civilian institutions according to a professional consultative body under Article 50 of the Veterinary Practice Act. In the area of water analysis, the Service cooperates with the Institute of Public Health and in the area of food of animal origin, it cooperates with the National Veterinary Institute - Institute for Food Hygiene.

Keywords: civil-military cooperation; dogs; epizoology; food;

forces; Service; Slovenian Armed Forces; veterinary

Prehosp Disast Med 2003;18(s1)s12.

\section{Federal Armed Forces School of Dog Handling}

Marion Hornkamp, MD

Federal Armed Forces School of Dog Handling, Germany

This paper provides an overview of the available training programs and veterinary services at the Federal Armed Forces School of Dog Handling.

Training programs prepare the dogs for the services they provide. These dogs are used to guard premises in Germany and are also deployed at military camps abroad.

Based on the dog's assigned role, specialty training is provided. At present, special canine training includes: (1) detection of explosives; (2) detection of drugs; (3) searchand-rescue efforts; and (4) explosive ordnance disposal (EOD dogs). These specially trained dogs are required for service in the various branches of the army (e.g., airborne infantry and the military police), and they are heavily employed during assignments abroad. Preparations for a pilot course to train mine-sniffing dogs currently are under way. In the future, service dogs earmarked for specialization will be trained in a new training center that has mock training scenarios set up in shelters to facilitate the entire range of canine specialty training.

The veterinary clinic includes facilities to provide preventive treatment and diagnostics and to treat sick animals. It is also responsible for purchasing new service dogs. The clinic is furnished with the latest medical equipment (instruments for x-ray, Doppler ultrasound, and endoscopy) and its staff includes a senior veterinarian specializing in small animals. The purchase of new service dogs is of paramount importance. In addition to their suitability, prospective dogs will be examined mainly with regard to their general health, skeleton structure, and teeth. X-ray examinations are done to check for any hip, elbow, and teeth defects. A special computer program is available to verify the given age of a dog on the basis of its tooth structure.

Veterinarians train every student handler in first aid and conduct preparatory courses for medical officers (veterinarian) scheduled for deployment abroad, which focus on 